\title{
Successful Treatment of Cavernous Sinus Metastasis from Follicular Thyroid Carcinoma with Lenvatinib
}

\author{
Tee Sin Lee ${ }^{1}$, Anna See ${ }^{2}$, Caleb Lim $^{3}$ and Xin Yong Huang ${ }^{1}$ \\ Department of Otorhinolaryngology Head \& Neck Surgery, Changi General Hospital, Department of Otorhinolaryngology \\ Head \& Neck Surgery, Singapore General Hospital ${ }^{2}, \mathrm{MOH}$ Holdings', Singapore
}

\begin{abstract}
Radioiodine-refractory distant metastases in well-differentiated thyroid carcinomas are rare but pose a significant diagnostic and management challenge. Follicular thyroid carcinoma which is a type of well-differentiated carcinoma, is known for its hematogenous spread. Various sites of distant metastasis have been described. We report the first-ever case of radioiodine-refractory metastasis at the cavernous sinus, arising from follicular thyroid carcinoma. The atypical site of metastasis and management complexity of radioiodine-refractory disease is discussed in this article.
\end{abstract}

Key Words: Follicular thyroid carcinoma, Metastases, Lenvatinib, Cavernous sinus

\section{Introduction}

Well-differentiated thyroid carcinoma is regarded as a highly curable cancer, associated with a 10-year survival rate ranging from 80 to $95 \% .^{1)}$ However, survival rates decrease by up to $50 \%$ if distant metastatic disease is present. ${ }^{2}{ }^{2}$ Distant metastases of differentiated thyroid carcinomas (papillary and follicular) can be found either at the time of diagnosis or after thyroidectomy, often through the use of radioiodine-uptake scans. Non-radioiodine avid distant metastases are rare and post a significant diagnostic and therapeutic challenge to clinicians. Loss of radioiodine avidity occurs when dedifferentiation occurs and is associated with a poorer outcome." We describe an extremely rare case of radioiodine-refractory cavernous sinus metastasis from follicular thyroid carcinoma which was successfully treated with the ty- rosine kinase inhibitor Lenvatinib. We explore in detail the complexity of this case which is contributed by i) an atypical site of metastasis and ii) the challenge of a managing a non-radioiodine avid metastasis.

\section{Case Report}

A 67-year-old male patient presented with a $7 \mathrm{~cm}$ thyroid nodule showing "follicular neoplasm" (Bethesda IV) on fine needle aspiration cytology. He underwent total thyroidectomy and histology returned as oncocytic variant of follicular thyroid carcinoma, with more than 10 foci of angoinvasion. He received standard adjuvant radioiodine ablation therapy with 106.3 $\mathrm{mCi}$ of lodine-131 (I-131). Concomitant whole-body radioiodine survey revealed no abnormal focal uptake of I-131 elsewhere.

Normal serum thyroglobulin antibody (TgAb) and undetectable thyroglobulin $(\mathrm{Tg})$ levels were recorded

Received April 28, 2020 / Revised May 16, 2020 / Accepted May 18, 2020

Correspondence: Anna See, MD, Department of Otorhinolaryngology Head \& Neck Surgery, Singapore General Hospital Outram Road, Singapore 169608

Tel: 65-6222-3222, Fax: 65-6224-9221, E-mail: anna.see.xinyin@singhealth.com.sg

Copyright (c) the Korean Thyroid Association. All rights reserved.

(1) This is an open-access article distributed under the terms of the Creative Commons Attribution Non-Commercial License (http://creative(c) (1) \& 8 commons.org/licenses/by-nc/4.0/), which permits unrestricted non-commercial use, distribution, and reproduction in any medium, provided the original work is properly cited. 
after surgery and surveyed every 6 months. He was commenced on high-dose oral levothyroxine for hormone replacement and thyroid-stimulating hormone (TSH) suppression. However, on regular surveillance, rising serum $\mathrm{Tg}$ values were noted. At $6^{\text {th }}$ month post-surgery, serum Tg was 79 micrograms/liter. At the end of 12 months, serum $\mathrm{Tg}$ had risen to $698 \mathrm{mi}-$ crograms/liter (normal, 2.0-70.0). A repeat dose of 30 $\mathrm{mCi}$ I-131 was administered under levothyroxine withdrawal which showed no evidence of radioiodine-avid disease. In view of the rising Tg with negative radioiodine scan, he was investigated with full-body positron emission tomography (PET) scan, administered under $8.6 \mathrm{mCi}$ of $\mathrm{F}-18$ fluorodeoxyglucose (FDG), which showed intensely FDG-avid bilateral thyroid beds lesions and multiple metastases at the left scapula, thoracic vertebrae and right sacrum. In addition, intensely FDG-avid soft tissue lesions in the left cavernous sinus and bilateral sphenoid sinuses were noted (Fig. 1).

Notwithstanding the earlier performed PET scan, a dedicated computed tomography scan of paranasal sinuses with contrast was undertaken, showing a 2.1 $\mathrm{cm}$ irregular mass the left cavernous sinus, abutting the left cavernous internal carotid artery (Fig. 1). Discussion at multidisciplinary tumor board recommended biopsy via the transsphenoidal approach. Histology returned as metastatic oncocytic follicular thyroid carcinoma, negative for BRAF V600E mutation. A repeat serum $\mathrm{Tg}$ level was markedly elevated at 3112 micrograms/liter. Two weeks later, he developed acute binocular diplopia, left retroorbital pain and proptosis. Ophthalmological examination showed left third and fourth cranial nerve palsies but negative relative afferent pupillary defect. Left cranial nerves VI, V and II were intact. A diagnosis of left cavernous sinus syndrome was made. Repeat multidisciplinary tumor board recommended urgent palliative radiotherapy and tyrosine kinase inhibitor (TKI) treatment with Lenvatinib. However, the patient was unable to tolerate radiotherapy and developed vomiting, headache and syncopal episodes. Neurosurgical intervention was discussed and deemed inappropriate as it conferred a high risk of blindness and cerebrospinal fluid leak. He was then placed on Lenvatinib therapy (14 micrograms once daily) and tolerated the treatment well with no gastrointestinal, cardiovascular, renal or dermatological side effects.

At the end of four weeks, the patient's left eye deficits had resolved completely with a corresponding remarkable radiological response on PET scan (Fig. 2). His osseous metastatic lesions did not show significant resolution. The serum Tg level was 422 micrograms/liter. At three years post-treatment, he remains visually asymptomatic and is gainfully employed as a private hire car driver.

\section{Discussion}

Metastases to the cavernous sinus are rare but have been described in solid carcinomas of the lung, colon, and liver. ${ }^{4,5)}$ Head and neck cancers have also been
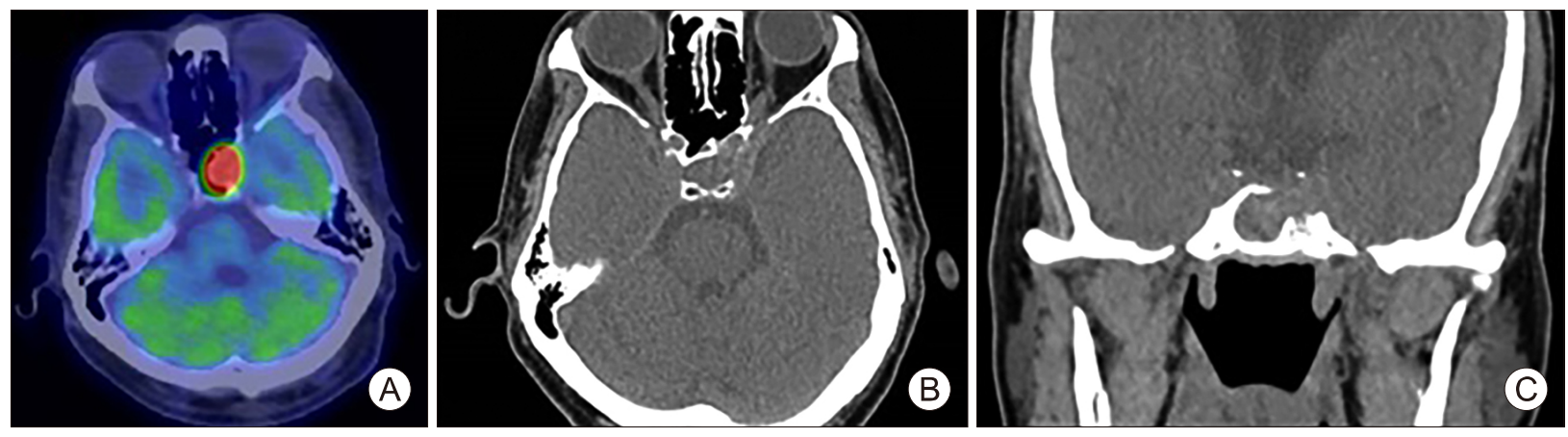

Fig. 1. (A) PET-CT showing an intensely FDG-avid lesion in the left cavernous sinus extending into the left sphenoid sinus, and the axial (B) and coronal (C) cuts of CT paranasal sinuses showing a soft tissue lesion in left cavernous sinus and bilateral sphenoid sinuses with bony erosion. 


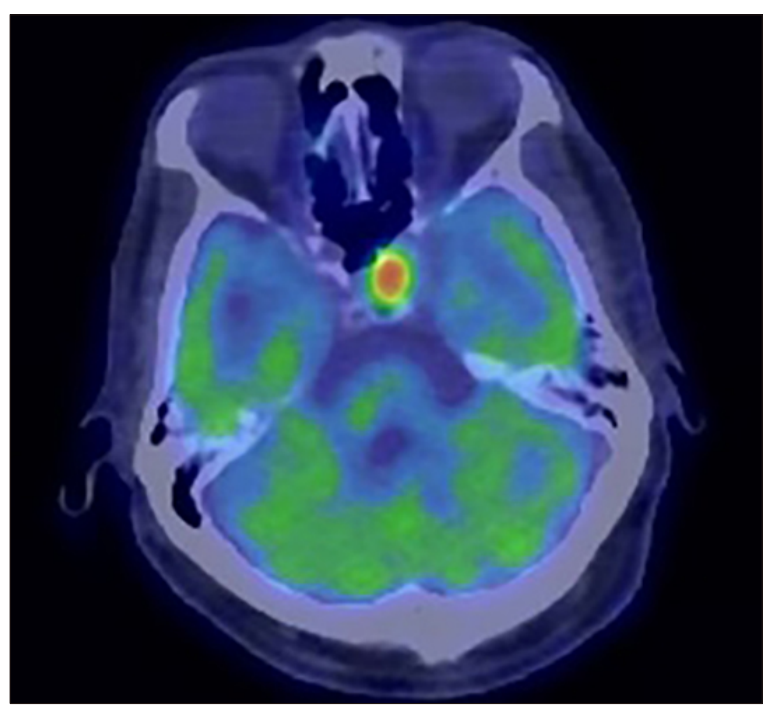

Fig. 2. Interval PET-CT after treatment with four weeks of Lenvatinib, showing significant reduction in size and FDG-avidity of metastasis.

reported to metastasize to the cavernous sinus. ${ }^{6)}$ Follicular thyroid carcinoma is a form of differentiated thyroid carcinoma with a known propensity for hematogenous spread and the most common sites of distant metastasis are pulmonary followed by osseous. When they occur in these sites, distant metastases are usually slow-growing and do not cause much symptoms. Consequently, watchful waiting is frequently adopted.

An extensive review of the English Literature on PubMed revealed that this is the first-ever reported case of follicular thyroid carcinoma with metastasis to the cavernous sinus and sphenoid sinus. An atypical location of metastasis to the cavernous sinus with debilitating functional deficits, coupled with radioiodine non-avidity, augmented the complexity of this case.

In the surveillance of thyroid carcinoma patients post-thyroidectomy, a combination of clinical examination, biochemistry and imaging are used. A rising serum $\mathrm{Tg}$ trend with no evidence of uptake on radioiodine scans should raise the suspicion of radioiodine-refractory disease. These are rare and the 10 -year survival rate is $10 \%$ from the time of detection of metastasis. ${ }^{7)}$

The advent of immunotherapy which targets vascular endothelial growth receptor (VEGFR) has expanded the therapeutic armamentarium for radio- iodine-refractory disease. Multitargeted TKIs have been investigated. In 2014, Sorafenib, a TKI that inhibits VEGFRs 1, 2, and 3, PDGFR $\beta$, Raf-1, RET, and BRAF, was approved by the U.S. Food and Drug Administration (FDA) for the treatment of radioiodine-refractory thyroid cancer based on results of a phase III trial showing a five-month improvement in median progression-free survival. ${ }^{8)}$

Similar to Sorafenib, Lenvatinib acts on VEGFRs 1 through 3, FGFRs 1 through 4, PDGFR $\alpha$, RET, and KIT signaling networks. ${ }^{9}$ A phase III trial (SELECT) was conducted to assess progression-free survival in patients with radioiodine-refractory thyroid cancer who received Lenvatinib versus those who received placebo. ${ }^{10)}$ Results showed that Lenvatinib was associated with significant improvement in the response rate $(64.8 \%$ in the Lenvatinib group vs. $1.5 \%$ in the placebo group; odds ratio, $28.87 ; 95 \% \mathrm{Cl}, 12.46$ to 66.86; $p<0.001)$. However, the authors also observed a significant number of side effects including diarrhea, hypertension, proteinuria and decreased appetite. ${ }^{10)}$ Our patient did not report these.

In conclusion, radioiodine-refractory disease should be promptly suspected in patients with rising serum Tg levels but negative radioiodine scans. While distant metastases in follicular thyroid carcinoma are usually regarded as slow-growing, atypical sites of metastases such as the cavernous sinus will require aggressive exploration of all available treatment modalities. Multitarget TKIs may prove to be a useful therapy for selected patients.

\section{Conflicts of Interest}

No potential conflict of interest relevant to this article was reported.

\section{Orcid}

Anna See: https://orcid.org/0000-0003-2126-4665

\section{References}

1) Gilliland FD, Hunt WC, Morris DM, Key CR. Prognostic 
factors for thyroid carcinoma. A population-based study of 15,698 cases from the Surveillance, Epidemiology and End Results (SEER) program 1973-1991. Cancer 1997;79(3):564-73.

2) Sampson E, Brierley JD, Le LW, Rotstein L, Tsang RW. Clinical management and outcome of papillary and follicular (differentiated) thyroid cancer presenting with distant metastasis at diagnosis. Cancer 2007;110(7):1451-6.

3) Riesco-Eizaguirre G, Gutierrez-Martinez P, Garcia-Cabezas MA, Nistal M, Santisteban P. The oncogene BRAF V600E is associated with a high risk of recurrence and less differentiated papillary thyroid carcinoma due to the impairment of $\mathrm{Na}+/ \mathrm{I}$ targeting to the membrane. Endocr Relat Cancer 2006;13(1): 257-69.

4) Mills RP, Insalaco SJ, Joseph A. Bilateral cavernous sinus metastasis and ophthalmoplegia. Case report. J Neurosurg 1981; 55(3):463-6.

5) Onec B, Oksuzoglu B, Hatipoglu HG, Onec K, Azak A, Zengin N. Cavernous sinus syndrome caused by metastatic colon carcinoma. Clin Colorectal Cancer 2007;6(8):593-6.

6) Zahra M, Tewfik HH, McCabe BF. Metastases to the cavernous sinus from primary carcinoma of the larynx. J Surg Oncol 1986;31(1):69-70.

7) Durante C, Haddy N, Baudin E, Leboulleux S, Hartl D, Travagli JP, et al. Long-term outcome of 444 patients with distant metastases from papillary and follicular thyroid carcinoma: benefits and limits of radioiodine therapy. J Clin Endocrinol Metab 2006;91(8):2892-9.

8) Brose MS, Nutting CM, Jarzab B, Elisei R, Siena S, Bastholt $\mathrm{L}$, et al. Sorafenib in radioactive iodine-refractory, locally advanced or metastatic differentiated thyroid cancer: a randomised, double-blind, phase 3 trial. Lancet 2014;384(9940):319-28.

9) Matsui J, Yamamoto Y, Funahashi Y, Tsuruoka A, Watanabe T, Wakabayashi T, et al. E7080, a novel inhibitor that targets multiple kinases, has potent antitumor activities against stem cell factor producing human small cell lung cancer H146, based on angiogenesis inhibition. Int J Cancer 2008;122(3):664-71.

10) Schlumberger M, Tahara M, Wirth LJ, Robinson B, Brose MS, Elisei R, et al. Lenvatinib versus placebo in radioiodinerefractory thyroid cancer. $N$ Engl J Med 2015;372(7):621-30. 